\title{
Formation and Destruction of Multilayered Tori in Coupled Map Systems
}

\author{
Zhusubaliyev, Zh. T.; Mosekilde, Erik
}

Published in:

Chaos

Link to article, DOI:

10.1063/1.2959141

Publication date:

2008

Document Version

Publisher's PDF, also known as Version of record

Link back to DTU Orbit

Citation (APA):

Zhusubaliyev, Z. T., \& Mosekilde, E. (2008). Formation and Destruction of Multilayered Tori in Coupled Map Systems. Chaos, 18(3), 037124. https://doi.org/10.1063/1.2959141

\section{General rights}

Copyright and moral rights for the publications made accessible in the public portal are retained by the authors and/or other copyright owners and it is a condition of accessing publications that users recognise and abide by the legal requirements associated with these rights.

- Users may download and print one copy of any publication from the public portal for the purpose of private study or research.

- You may not further distribute the material or use it for any profit-making activity or commercial gain

- You may freely distribute the URL identifying the publication in the public portal

If you believe that this document breaches copyright please contact us providing details, and we will remove access to the work immediately and investigate your claim 


\title{
Formation and destruction of multilayered tori in coupled map systems
}

\author{
Zhanybai T. Zhusubaliyev ${ }^{1, a)}$ and Erik Mosekilde ${ }^{2, b)}$ \\ ${ }^{1}$ Department of Computer Science, Kursk State Technical University, 50 Years of October Str., \\ 94, Kursk 305040, Russia \\ ${ }^{2}$ Complex Systems Group, Department of Physics, Technical University of Denmark, 2800 Lyngby, Denmark
}

(Received 21 December 2007; accepted 24 June 2008; published online 22 September 2008)

\begin{abstract}
The paper first illustrates how multilayered tori can arise through one or more pitchfork bifurcations of the saddle cycle on an ordinary resonance torus. The paper hereafter describes three different scenarios by which a multilayered torus can be destructed. One scenario involves a saddle-node bifurcation in which the middle layer of a three-layered torus disappears in an abrupt transition to chaos while the outer-layer manifolds and their associated saddle and unstable-focus cycles continue to exist and to control the transient dynamics. In a second scenario, the unstable focus cycles of the intermediate layers in a five-layered torus turn into unstable nodes, and closed loop connections are established between the unstable nodes and the points of the stable resonance node on the torus. Finally, a third scenario describes a transition in which homoclinic bifurcations destroy first the outer layers and thereafter also the inner layer. The paper also illustrates how the formation and destruction of multilayered tori can occur in the cluster dynamics of an ensemble of globally coupled maps. This leads to three additional scenarios for the destruction of multilayered tori.

(C) 2008 American Institute of Physics. [DOI: 10.1063/1.2959141]
\end{abstract}

Torus destruction represents one of the classic scenarios for the transition to chaos. This transition is typical of systems of coupled oscillators where it often replaces the route to chaos via a cascade of period-doubling bifurcations. In contrast to the well-described period-doubling and intermittency routes to chaos, torus destruction involves nonlocal bifurcations, and many aspects of the mechanisms underlying torus destruction still remain to be explored in detail. Descriptions of the torus destruction scenario typically start out from a pair of saddle and node cycles on a resonance torus and follow how the manifolds associated with these states start to fold, wind or intersect. We have found that a torus can develop several parallel sets of manifolds. This can occur, for instance, if the original saddle solution undergoes a pitchfork or period-doubling bifurcation transverse to the torus manifold. The paper describes the formation of three- and five-layered tori in a system of two coupled noninvertible maps and discusses different scenarios for the breakdown of such multilayered tori. The paper also considers an ensemble of globally coupled maps and show how multilayered tori can occur in the asymptotic dynamics of such systems as well. We suggest that the formation of multilayered tori is directly related to the noninvertibility of the examined maps.

\section{INTRODUCTION}

When two identical period-doubling systems are coupled symmetrically one often observes that the period-doubling transition to chaos is replaced by a transition through quasiperiodicity and torus destruction. Such a replacement was

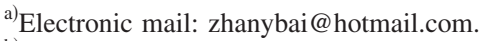

${ }^{b)}$ Electronic mail: Erik.Mosekilde@fysik.dtu.dk.
}

noticed, for instance, by Van Buskirk and Jeffries ${ }^{1}$ in experiments with driven electronic resonators. When varying the drive voltage, the single resonator showed a period-doubling transition to chaos, whereas a pair of resistively coupled resonators exhibited a transition through torus destruction. The reason why this replacement occurs, is that for diffusively coupled oscillators, a transverse period doubling precedes the normal symmetric period doubling at an early stage in the bifurcation cascade, and the produced antisymmetric period-2 cycle hereafter undergoes a Hopf bifurcation (or a Neimark-Sacker bifurcation for map systems). ${ }^{2-4}$

Afraimovich and Shilnikov ${ }^{5}$ have identified three different scenarios for the transition from quasiperiodicity to chaos for smooth, invertible maps. A description of these transitions starts from a smooth resonance torus defined by the union of a stable node cycle, the corresponding saddle cycle, and the unstable manifold of the saddle cycle. With increasing nonlinearity, this manifold begins to fold as it approaches the points of the node. In this way the smoothness of the torus is lost in the discrete points of the stable cycle. When the system under variation of a parameter crosses out of the resonance tongue, this nonsmoothness spreads to the entire structure, and the torus is destroyed. It is also possible that the node is transformed into a stable focus and the manifold winds around the focus points or that the unstable manifold starts to intersect the stable manifold of the saddle solution such that a Smale horseshoe structure is formed, leading again to the destruction of the torus and a transition to chaos.

In recent years, the Afraimovich-Shilnikov theory has been extended to describe some of the modifications that can arise for noninvertible maps ${ }^{6-9}$ and for piecewise-smooth systems. In the first case, one can observe that the folding and winding manifolds produce self-intersecting loops, ${ }^{10}$ and in the latter case the mechanism of torus destruction can 


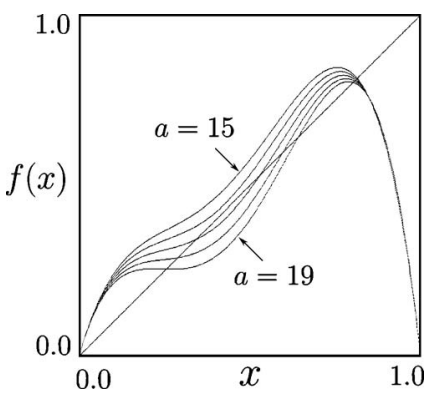

FIG. 1. Variation of the map $f(x)$ for different values of $a$ ranging from $a=15$ to $a=19$.

involve border-collision bifurcations where the multipliers of an orbit suddenly jump from one position in the complex plane into another. ${ }^{11,12}$

The purpose of the present study is to examine the formation and destruction of tori that are formed as layered structures of several sets of parallel manifolds each with their associated stable or unstable resonance modes. Such tori can arise, for instance, if the saddle cycle on a resonance torus undergoes one or more period-doubling or pitchfork bifurcations transversely to the torus. We have recently observed the formation of double-layered tori in connection with the analysis of a dc/dc converter with two-zone pulse-width modulated control. ${ }^{12}$ The present paper first considers a system of two linearly coupled, identical one-dimensional maps:

$$
f:\left(\begin{array}{l}
x \\
y
\end{array}\right) \mapsto\left\{\begin{array}{l}
f(x)+\varepsilon(y-x) ; \\
f(y)+\varepsilon(x-y),
\end{array}\right.
$$

where $f(x)=x(1-x)\left[a x^{2}+\left(b^{2}-d a\right) x+c\right]$ and $\varepsilon$ is the coupling parameter. The individual map $f(x)$ is illustrated in Fig. 1. Here, we have chosen $b^{2}=2.85, c=3.5$, and $d=0.85$. In the following bifurcation analysis, we shall use the coupling parameter $\varepsilon$ and the nonlinearity parameter $a$ as control parameters.

The map $f(x)$ represents the canonical map for discussions of transitions to chaos via type I intermittency. Transitions of this type occur in practical all systems that display deterministic chaos, and the saddle-node bifurcations leading, for instance, to the formation of the characteristic periodic windows in the logistic map are all transitions from intermittency I to regular periodic dynamics. Transitions to chaos via type I intermittency have historically played a significant role in discussions of the onset of turbulence ${ }^{13,14}$ and they continue to be the main route to chaos in many biological models. ${ }^{15,16}$

For $16.5<a<22.1, f(x)$ displays four fixed points of which the fixed point in $x=0$ is unstable for the parameters of interest in the present study. The fixed point near the top of the map arises in a saddle-node bifurcation for $a \approx 22.1$ and remains stable until $a \approx 16.1$, where it undergoes the first of a cascade of period-doubling bifurcations.

For $a>16.5, f(x)$ displays two intermediate fixed points of which the lower fixed point for the considered values of $a$ is a node and the upper a saddle. At $a \approx 16.5$, these two fixed points merge and disappear in a tangent bifurcation, and in a certain interval of $a$ the map hereafter displays type I

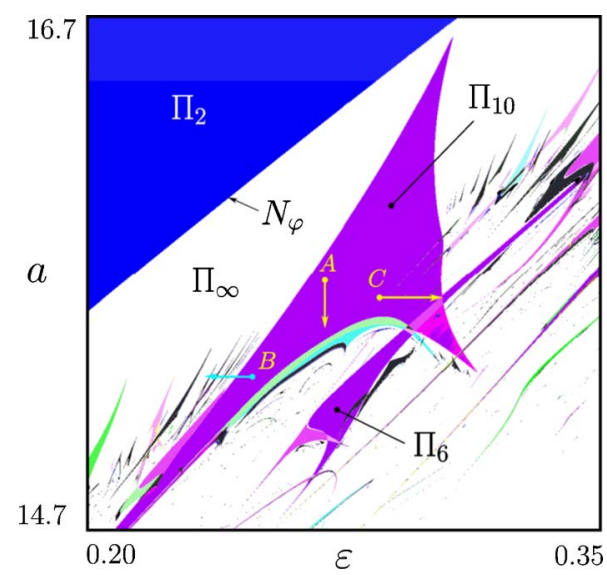

FIG. 2. (Color online) Chart of the dynamical modes in the parameter plane $(\varepsilon, a) . \Pi_{2}$ is the domain of existence of the stable period- 2 cycle. $\Pi_{6}$ and $\Pi_{10}$ are the regions of period- 6 and period-10 resonant dynamics, respectively. $N_{\varphi}$ is the Neimark-Sacker bifurcation boundary and $\Pi_{\infty}$ is the domain of quasiperiodicity. In the lower right part of the figure, the white region, interrupted by narrow resonance zones, denotes the occurrence of deterministic chaos.

intermittency. ${ }^{13,14}$ The coupled map system (1) has previously been used ${ }^{17}$ to study chaotic synchronization and riddled basins of attraction ${ }^{18-20}$ for coupled intermittency maps. The idea of the present study is to follow the transitions that occur for the transverse period-2 node $N$ $=\left(N_{1}, N_{2}\right)$ as the parameters $a$ and $\varepsilon$ are changed. This leads us to describe the formation of tori with both three and five layers of parallel manifolds and to consider three different mechanisms for the breakdown of such tori. We also show that multilayered tori can arise in the symmetric and asymmetric two-cluster dynamics for a system of many coupled intermittency maps. In this case, yet a different set of mechanisms of multilayered-torus breakdown are active.

\section{BIRTH OF A THREE-LAYERED TORUS}

As long as the coupling parameter $\varepsilon$ is small enough, the map (1) has two stable fixed points on the diagonal $x=y$. When the value of $\varepsilon$ is increased, the top most fixed point $N_{0}$ becomes transversally unstable and undergoes a supercritical transverse period doubling. This produces a stable period-2 cycle $N=\left(N_{1}, N_{2}\right)$ transverse to the diagonal $x=y$. In accordance with the above discussion, ${ }^{2-4}$ the transverse period doubling occurs before the synchronized period doubling. Moreover, the symmetric period- 2 cycle arising in the synchronous bifurcation is born as a saddle cycle, and the next bifurcation to take place for the asymmetric period-2 cycle is a Neimark-Sacker bifurcation.

Figure 2 shows a chart of the dynamical modes in the $(\varepsilon, a)$ parameter plane for the map (1). $\Pi_{2}$ denotes the region of existence for the stable asymmetric period- 2 cycle, and $N_{\varphi}$ is the Neimark-Sacker bifurcation curve along which the period-2 cycle is transformed into a two-band quasiperiodic attractor. The region of quasiperiodicity is denoted $\Pi_{\infty}$. In this region we find the usual dense set of resonance tongues, with $\Pi_{6}$ and $\Pi_{10}$ representing two of the most prominent tongues. In these tongues the system displays period- 6 and period-10 dynamics, respectively. In the lower right part of 


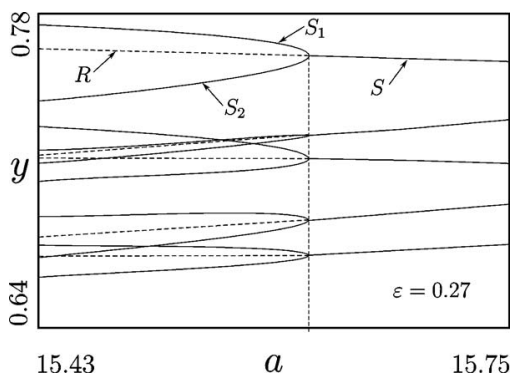

FIG. 3. Birth of the three-layered torus through a pitchfork bifurcation as the nonlinearity parameter $a$ is reduced through the bifurcation point at $a \approx 15.62 . S, S_{1}$, and $S_{2}$ are period-10 saddle cycles, and $R$ is an unstable node period-10 cycle (the repeller). Since the map is symmetric with respect to the diagonal $y=x$ we only show five points, belonging to the lower right branch, for each of the three saddle cycles. When the bifurcation occurs, the stable period-10 cycle has turned into a focus cycle (Fig. 4).

the figure, the white region, interrupted by narrow resonance zones, denotes the occurrence of deterministic chaos.

Let us first examine the transition that occurs as we move along the direction $A$ in the resonance tongue $\Pi_{10}$, i.e., as we reduce the parameter $a$ from 15.75 to 15.43 while maintaining the coupling parameter constant at $\varepsilon=0.27$. This transition is shown in Fig. 3. At the starting point, i.e., for $a=15.75$, the map (1) displays an invariant torus that is the union of the unstable manifold of the period-10 saddle cycle $S$, the saddle cycle itself, and the points of the stable period-10 node $N$. Since the map is symmetric with respect to the diagonal $x=y$, the figure only shows the five saddle points $S$ that belong to one branch of the torus. As $a$ is reduced, the stable node $N$ first turns into a focus $F_{1}$ (as its multipliers become complex), and the unstable manifolds of the saddle start to wind around the stable cycle. This is illustrated in the phase portrait in Fig. 4. At this stage, the torus is no longer smooth in the points of the period- 10 focus $F_{1}$. At $a \approx 15.62$ the saddle period- 10 cycle $S$ undergoes a pitchfork bifurcation. The original saddle cycle $S$ turns into a repellor (an unstable node), and two new saddle period-10 cycles $S_{1}$ and $S_{2}$ appear (Fig. 3). As a result, a three-layered torus structure softly arises from the original resonance torus.

Figure 5 presents the phase portrait of the map for the fully developed three-layered torus. A magnified part of this

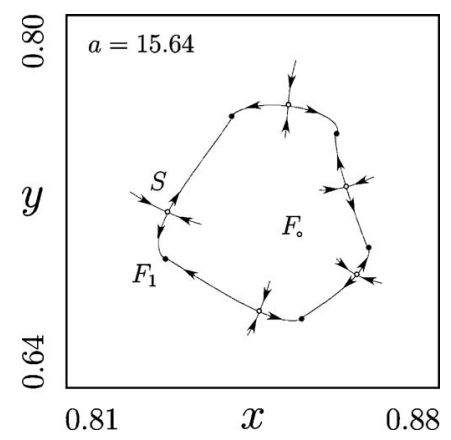

FIG. 4. Phase portrait of the map immediately before the pitchfork bifurcation in Fig. 3. $\varepsilon=0.27$ and $a=15.64$. $S$ represents the points of the period-10 saddle cycle and $F_{1}$ the points of the stable resonance cycle. This cycle has now turned into a focus, and, although this is difficult to see in the figure, the unstable manifold from the saddle cycle winds around the points of the stable cycle.

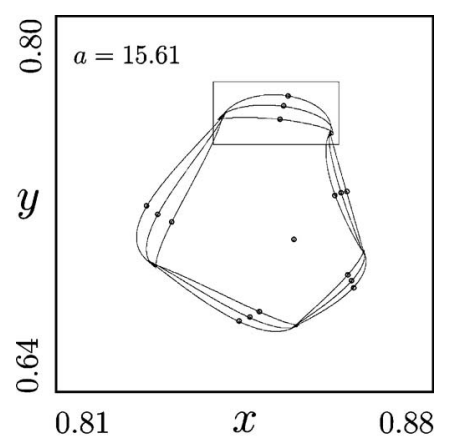

(a)

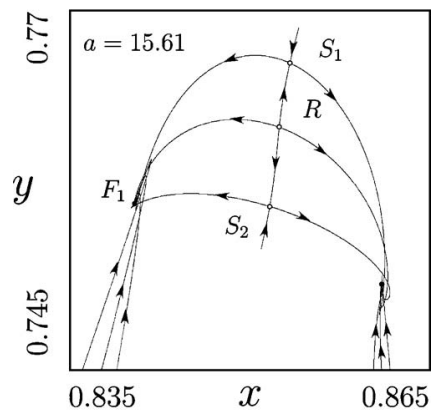

(b)

FIG. 5. Birth of a three-layered torus through the pitchfork bifurcation of the saddle cycle. (a) Phase portrait for the fully developed three-layered torus. $\varepsilon=0.27$ and $a=15.61$. (b) Magnified part of the phase portrait that is outlined by the rectangle in (a). Here $F_{1}$ is a stable period-10 focus cycle.

phase portrait, outlined by the rectangle in Fig. 5(a), is shown in Fig. 5(b). The external layers of the three-layered torus are formed by the unstable manifolds of the saddlecycles $S_{1}$ and $S_{2}$. Such a closed invariant curve is often referred to as a saddle-stable focus connection. ${ }^{21}$ The middle layer is repelling and is composed by the nonleading unstable manifold of the unstable node period-10 cycle $R$.

\section{FORMATION AND DESTRUCTION OF A FIVE-LAYERED TORUS}

Let us now examine how the three-layered torus is transformed into a five-layered torus to finally be destroyed as we leave the resonance tongue $\Pi_{10}$ in the direction $B$ (see Fig. 2 ). We have observed several different scenarios for this transformations. They will be illustrated through a series of detailed, numerically calculated phase portraits and bifurcation diagrams that show both the formation of loop tori, the formation of a five-layered torus, and the processes through which the multilayered torus is finally destroyed.

First, the external layers of the three-layered torus transform into loop tori as infinite sequences of self-intersecting loops arise along the unstable manifold of the saddle-cycles near the stable node. ${ }^{7}$ The middle layer also turns nonsmooth due to folding of the unstable manifold near the stable node but without intersections. As an example, Fig. 6 shows the phase portrait of the map for $\varepsilon=0.2437$ and $a=15.3$ after the transformation from nonsmooth torus to loop or cusp torus. The mechanisms of transition from the nonsmooth torus to loop torus were described in detail by Maistrenko et al. ${ }^{10}$ 


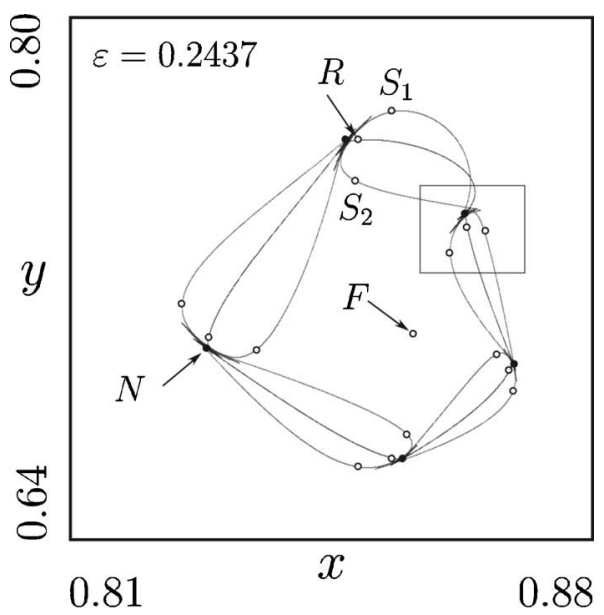

(a)

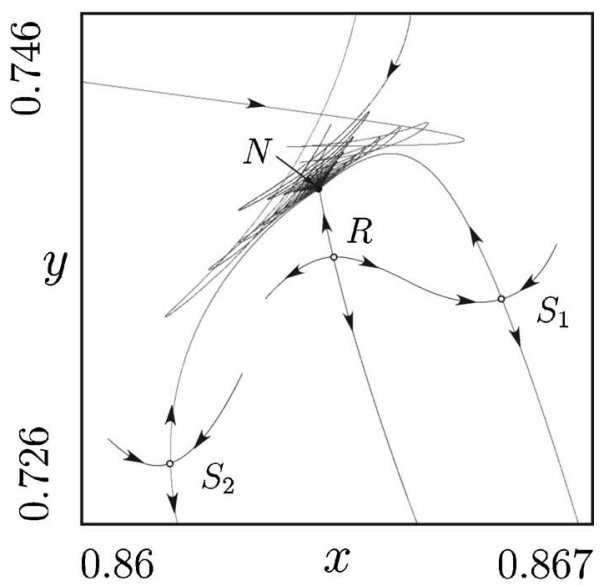

(b)

FIG. 6. Three-layered torus before the second pitchfork bifurcation. $\varepsilon=0.2437$ and $a=15.3$. Magnification of part of the phase portrait (b). Note how the manifolds of the three-layered torus fold as they approach the points of the stable node. For the two outer layers, this folding produces a loop or cusp torus (Ref. 10).

As a next step in the transformation, the unstable node $R$ undergoes a new pitchfork bifurcation as one of its multipliers passes through +1 and becomes less than +1 . In this bifurcation, as illustrated in Fig. 7, two unstable nodes, the repellors $R_{1}$ and $R_{2}$, softly arise from the unstable node $R$, and the unstable node $R$ becomes a saddle $S_{0}$. This bifurcation leads to the birth of a five-layered torus.

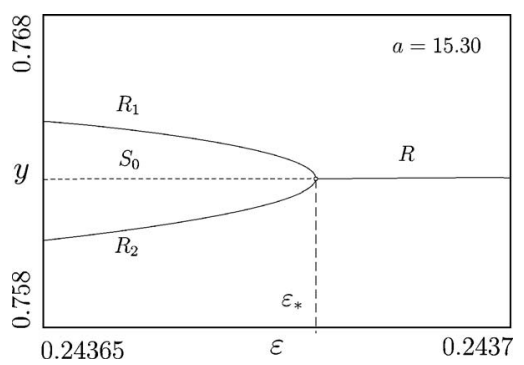

FIG. 7. Birth of the five-layered torus through the pitchfork bifurcation that occurs as the coupling parameter is reduced through the bifurcation point $\varepsilon_{*}$. $R, R_{1}$, and $R_{2}$ are the unstable node period-10 cycles (the repellors), $S_{0}$ is the saddle period- 10 cycle.

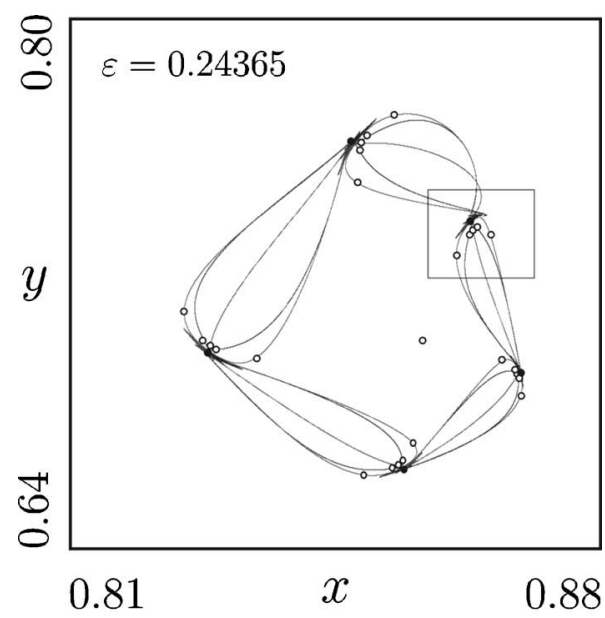

(a)

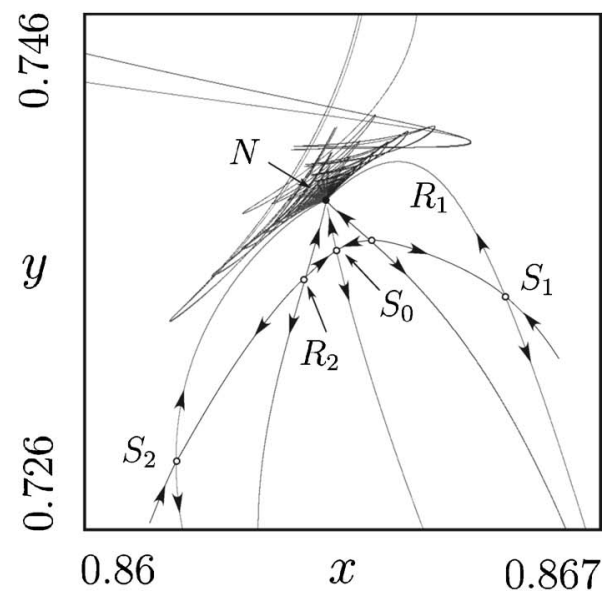

(b)

FIG. 8. (a) The fully developed five-layered torus. $\varepsilon=0.24365$ and $a=15.3$. (b) Magnified part of the phase portrait that is outlined in (a) by the rectangle.

Figure 8 shows the phase portrait of the map after the pitchfork bifurcation for $\varepsilon=0.24365$ and $a=15.3$. The magnified part of the torus outlined by the rectangle in Fig. 8(a) is shown in Fig. 8(b). The middle and two external layers of this torus are attracting. These layers are composed by the unstable manifolds of the saddle cycles $S_{0}, S_{1}$, and $S_{2}$. The two intermediate layers, formed by the unstable manifolds of two unstable nodes $R_{1}$ and $R_{2}$, are repelling.

With further change of parameter $a$ along the direction $B$, one can observe the reverse transition from a five-layered torus to a three-layered torus. As illustrated in the phase portrait of Fig. 9, this transition involves the transformation of the unstable nodes $R_{1}$ and $R_{2}$ in Fig. 8 into the unstable focus cycles $F_{1}$ and $F_{2}$, as the real multipliers of the unstable nodes become complex. As a result the two intermediate layers disappear, and we are left with a three-layered torus with two embedded focus cycles.

In the first scenario to be presented, the three-layered torus is destroyed through a saddle-node bifurcation: the stable node $N$ and the saddle $S_{0}$ merge and disappear as we leave the resonance tongue $\Pi_{10}$. Our numerical experiments indicate that this leads to an abrupt transition from periodic 


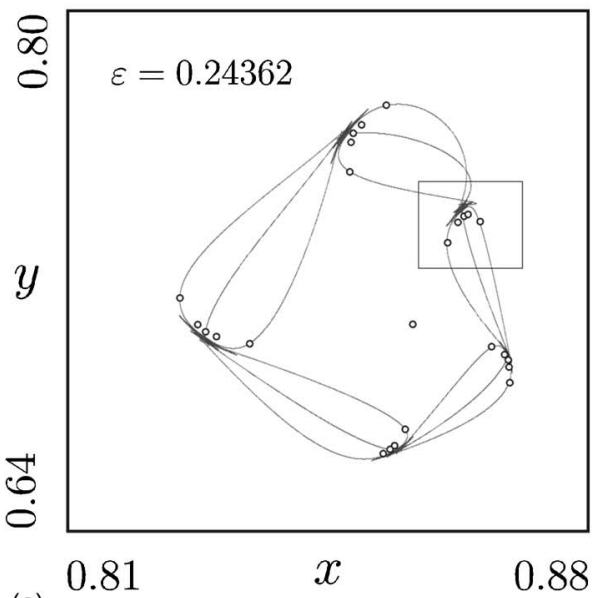

(a)

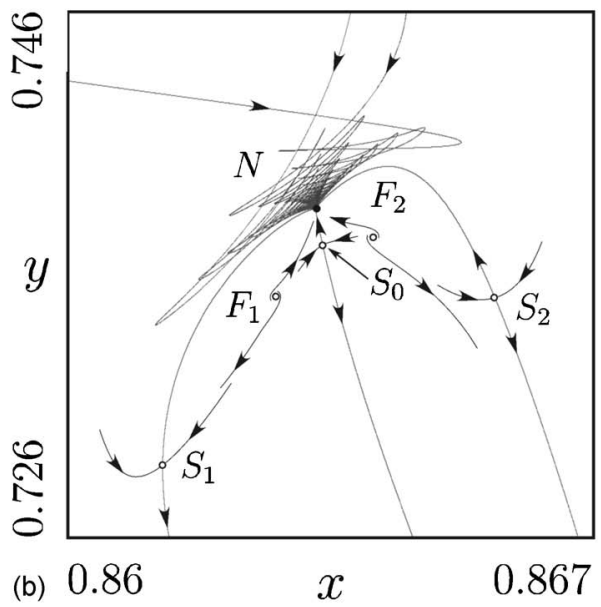

FIG. 9. (a) Phase portrait of the map after destruction of the two intermediate layers through transformation of the unstable nodes $R_{1}$ and $R_{2}$ into unstable focus cycles. $\varepsilon=0.24362$ and $a=15.3$. (b) Magnified part of the phase portrait outlined by the rectangle in (a).

to chaotic oscillations. Figures 10-12 illustrate the main stages of this process when the parameter $a$ is changed along the direction $B$ in the region $\Pi_{10}$.

Figures 10 and 11 show the phase portrait of the map immediately before and in the point of saddle-node bifurcation at the edge of the resonance tongue. Further change of the parameter $a$ leads to the appearance of chaotic oscillations. Figure 12 illustrates the phase portrait of the map after the torus destruction and the transition to chaos. In the numerical experiments we observe a hard transition from periodic to chaotic oscillations. Note that the two saddle cycles $S_{1}$ and $S_{2}$ continue to exist, and the same is true for the focus cycles $F_{1}$ and $F_{2}$. As shown in Fig. 12, the unstable manifolds associated with these cycles are drawn into the chaotic set.

\section{ALTERNATIVE MECHANISMS FOR THE DESTRUCTION OF MULTILAYERED TORI}

In our second scenario, the five-layered torus is destroyed through the formation of a three-layered torus with an "unstable node-stable node" structure. The five-layered torus first transforms into a three-layered torus as described

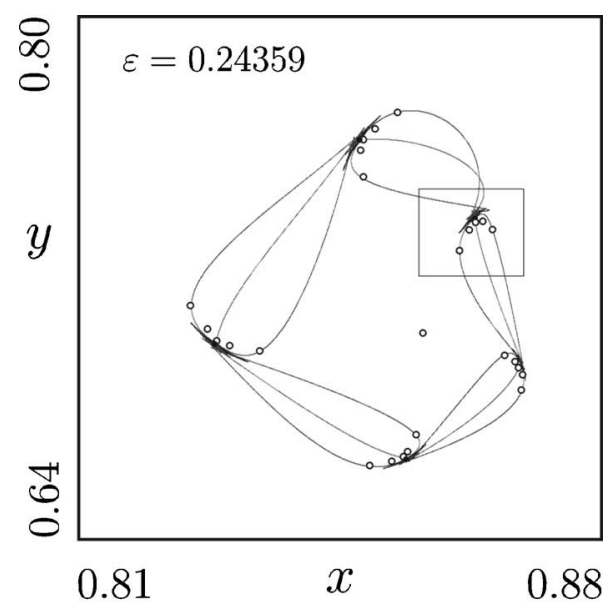

(a)

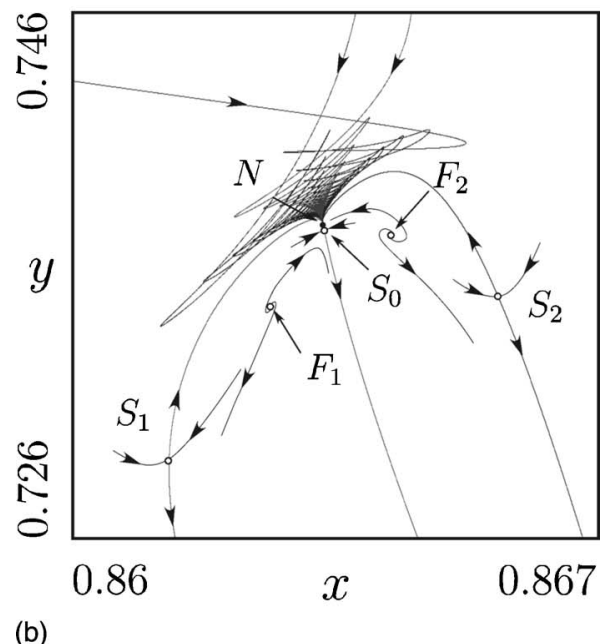

FIG. 10. (a) Phase portrait of the map near the saddle-node bifurcation boundary of the resonance zone $\Pi_{10} . \varepsilon=0.24359$, and $a=15.3$. (b) Magnified part of the phase portrait outlined by the rectangle in (a).

in Figs. 8 and 9, i.e., the unstable node cycles $R_{1}$ and $R_{2}$ turn into the unstable focus cycles $F_{1}$ and $F_{2}$ (see, for example, Fig. 9) as the real multipliers of the unstable nodes become complex. This leads to the destruction of the two intermediate layers. Hereafter, the unstable focus cycles $F_{1}$ and $F_{2}$ transform into unstable nodes $R_{1}$ and $R_{2}$ (see Fig. 13) as their the multipliers again become real. This leads to the formation of unstable node-stable node loops. Figures 13 and 14 illustrate the phase portraits of the map after this transition. Figure 13 shows a three-layered torus with "unstable nodestable node" loops, and Fig. 14 illustrates these loops separately.

As we can see from Fig. 14, the nonleading manifolds of the unstable nodes $R_{1}$ and $R_{2}$ form loops that fold near the points of the stable period-10 cycle. Finally, the unstable nodes $R_{1}$ and $R_{2}$ merge with the saddles $S_{1}$ and $S_{2}$ and disappear. This leads to the destruction of the two external layers. However, the middle layer continues to exist. When we leave the resonance tongue, this layer is destroyed through a saddle-node bifurcation according to the classic scenario.

In a third scenario, which is observed if we leave the resonance tongue $\Pi_{10}$ along the direction $C$, the three- 


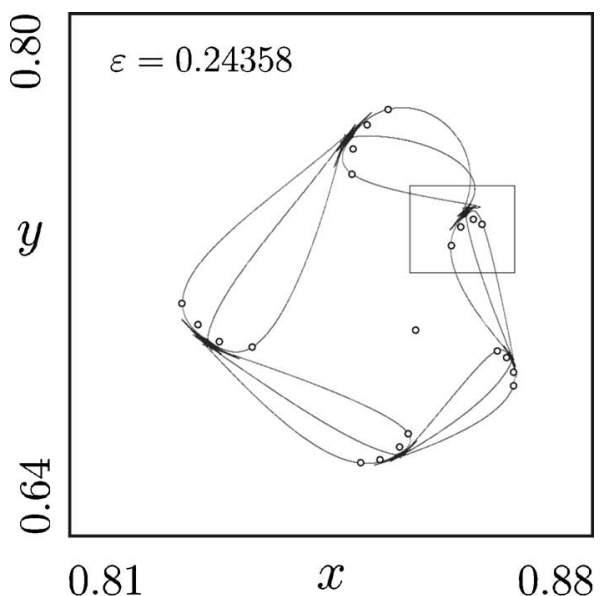

(a)

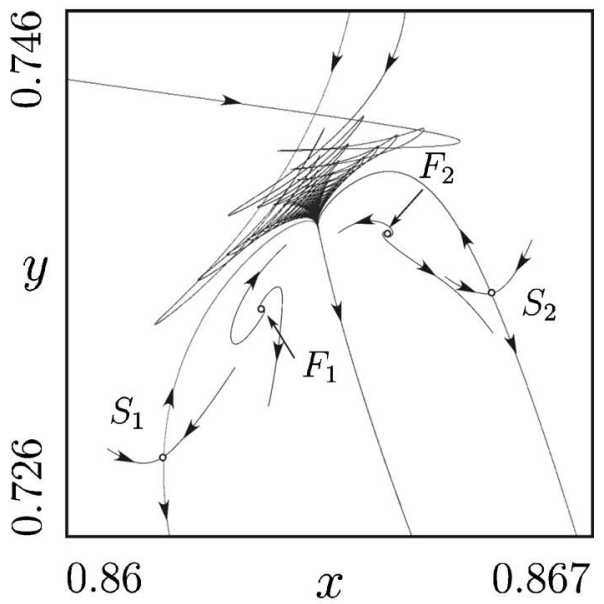

(b)

FIG. 11. (a) Phase portrait of the map at the saddle-node bifurcation. $\varepsilon=0.24358$ and $a=15.3$. (b) Magnified part of the phase portrait outlined by the rectangle in (a).

layered torus is destroyed through a homoclinic bifurcation in the following way: first, two external layers are destroyed when the stable and unstable manifolds of the saddle periodic orbit become tangent to each other. The middle layer continues to exist. Finally, the middle layer is destroyed in a similar manner. Figures 15 and 16 demonstrate the main stages of this process.

Figure 15(a) shows the phase portrait of the map before the first homoclinic tangency. The external layers of this torus are formed by the a saddle-stable focus connection. These layers contain loops [Figs. 15(b) and 16(a)]. The middle layer is composed by the nonleading unstable manifold of the unstable node period-10 cycle. Before its destruction the middle layer transforms from a nonsmooth torus to a loop torus via the usual cusp torus. ${ }^{10}$

\section{MULTILAYERED TORI IN ENSEMBLES OF GLOBALLY COUPLED MAPS}

Let us hereafter consider an ensemble of $N$ globally coupled, identical maps, ${ }^{22-24}$

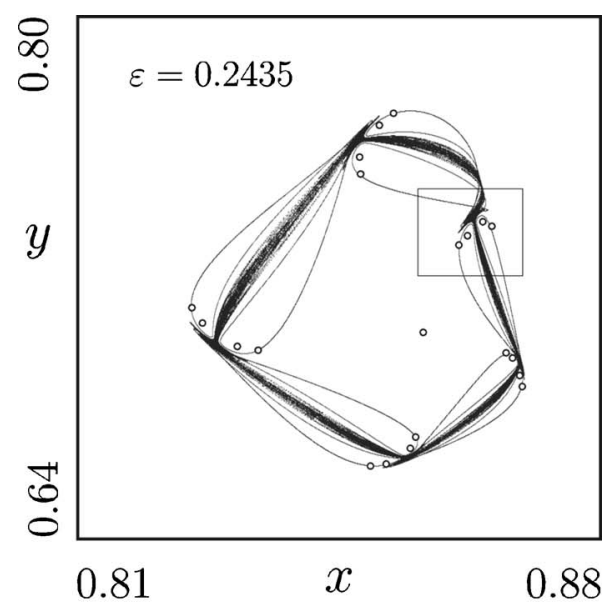

(a)

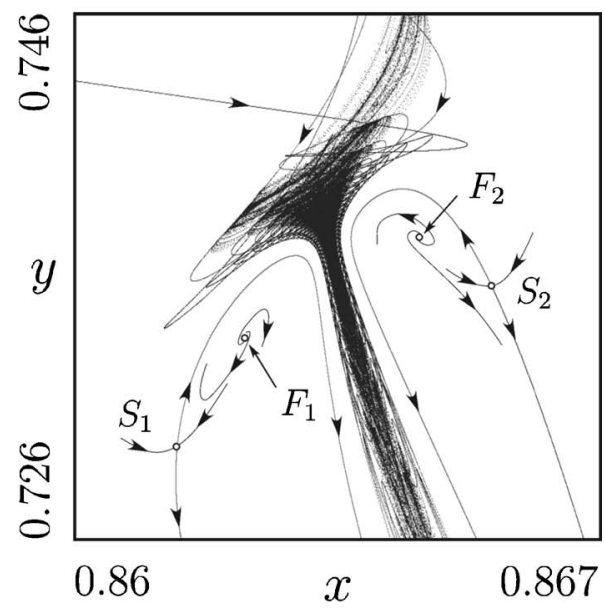

(b)

FIG. 12. (a) Phase portrait of the map after the torus has been destroyed $\varepsilon=0.2435$ and $a=15.3$. (b) Magnified part of the phase portrait outlined by the rectangle in (a). Note that both the saddle cycles $S_{1}$ and $S_{2}$ and the focus cycles $F_{1}$ and $F_{2}$ continue to exist.

$x_{k}(i)=(1-\varepsilon) f\left[x_{k-1}(i)\right]+\frac{\varepsilon}{N} \sum_{j=1}^{N} f\left[x_{k-1}(j)\right], \quad k=1,2, \ldots$,

where $k$ is a discrete time step and $i$ represents the number of a particular oscillator in the ensemble $(i=1,2, \ldots, N) . x_{k}(i)$ describes the dynamics of the individual map, and $\varepsilon$ is the coupling constant.

The concept of an ensemble of globally coupled oscillators is complementary to the concept of coupled map lattices (CMLs). ${ }^{25}$ Where the oscillators in a coupled map lattice are considered to be arranged in a regular spatial structure and to interact with their immediate neighbors, an ensemble of globally coupled maps disregards the spatial organization and considers all oscillators to interact with each other. In the biological realm, a CML may be used, for instance, to model interacting muscle cells in the heart ${ }^{26}$ or interacting insulin producing cells in the pancreas ${ }^{27}$ where local coupling via gab-junctions between the cells causes different forms of electrochemical waves to propagate across the tissue. An ensemble of globally coupled oscillators, on the other hand, can be used to model interacting nerve cells in the brain where 


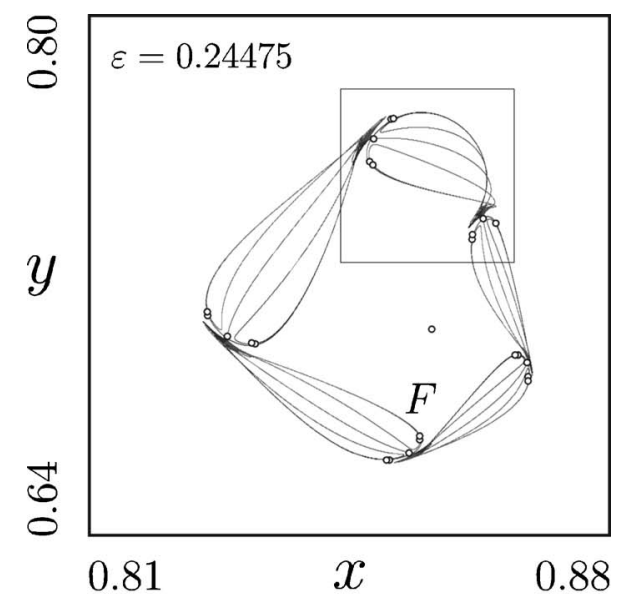

(a)

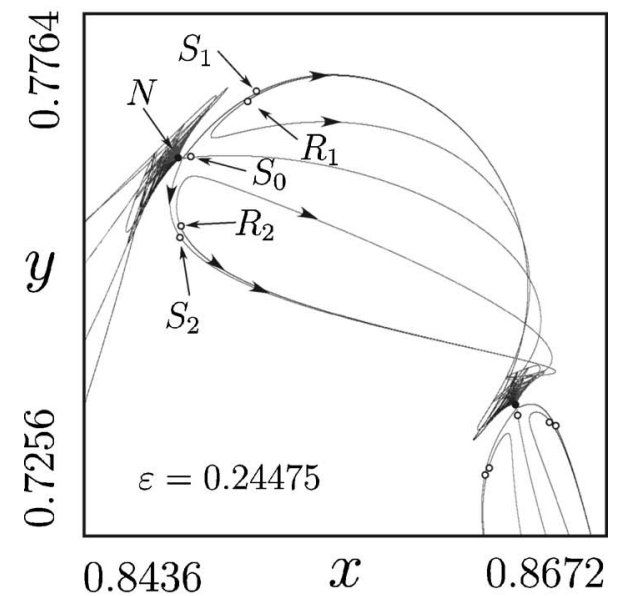

(b)

FIG. 13. Three-layered torus with the "unstable node-stable node" loops. $\varepsilon=0.24475$ and $a=15.3$. (b) Magnified part of the phase portrait outlined by the rectangle in (a).

synchronization of the spiking dynamics for clusters of cells is thought to represent the problem underlying Parkinsonian and other forms of tremor. ${ }^{28}$

In such ensembles, the multimode dynamics of the uncoupled system is often suppressed by a tendency of the oscillators to entrain. For certain values of the parameters $a$ and $\varepsilon$, the state of full synchronization may attract most trajectories. For other parameter values, however, the ensemble may split up in two or more clusters such that oscillators within a given cluster operate in synchrony, but not in synchrony with oscillators in other clusters. Two-cluster dynamics, for instance, may be described by the equations

$x_{k}=f\left(x_{k-1}\right)+p \varepsilon\left[f\left(y_{k-1}\right)-f\left(x_{k-1}\right)\right]$;

$y_{k}=f\left(y_{k-1}\right)+(1-p) \varepsilon\left[f\left(x_{k-1}\right)-f\left(y_{k-1}\right], \quad k=1,2, \ldots\right.$,

$f(x)=x(1-x)\left[a x^{2}+\left(b^{2}-d a\right) x+c\right]$

where $p(p \in[0,1])$ defines the distribution of oscillators between the two clusters. If $N_{1}$ oscillators in the ensemble synchronize in one asymptotic state and the remaining $N_{2}=N$ $-N_{1}$ synchronize in another state, then $p=N_{1} / N_{2}$. For sufficiently large values of $N, p$ can be considered as a pseudo-

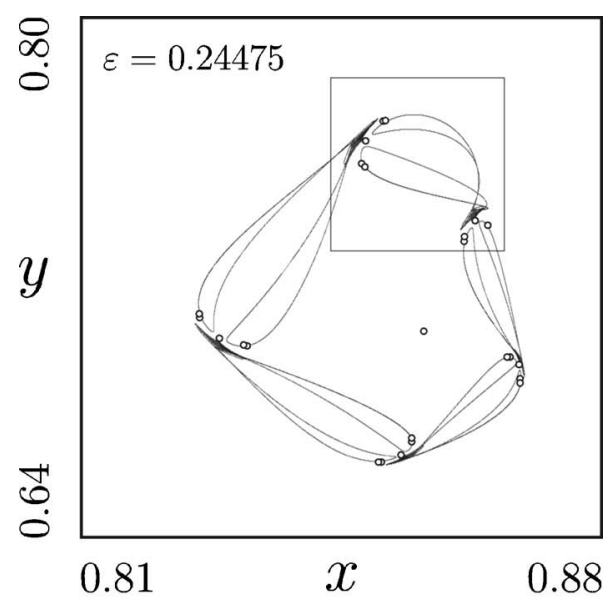

(a)

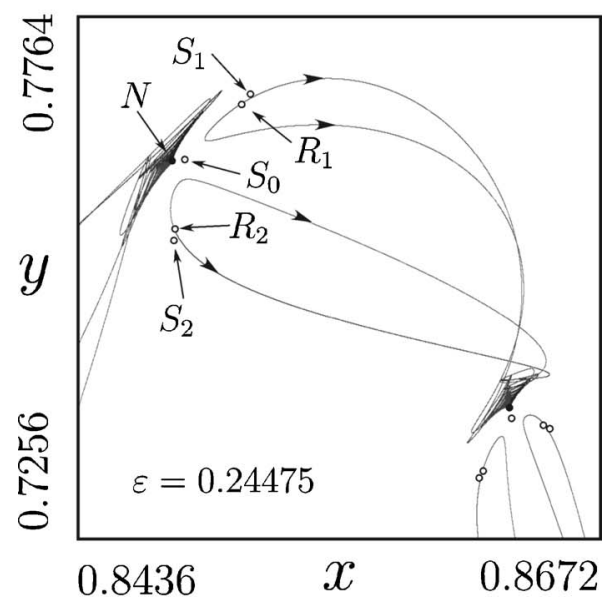

(b)

FIG. 14. "Unstable node-stable node" loop. $\varepsilon=0.24475$ and $a=15.3$. (b) Magnified part of the phase portrait outlined by the rectangle in (a).

continuous variable, and the absolute number of oscillators no longer matters for a description of the asymptotic dynamics of the ensemble. $p=0.5$ corresponds to a symmetric cluster splitting and $p \neq 0.5$ represents an asymmetric splitting. In general, one does not expect the behavior of the system to be critically dependent on small asymmetries, ${ }^{24}$ except, of course, that a pitchfork bifurcation in the symmetric case $(p=0.5)$ may be replaced by a saddle-node bifurcation in the asymmetric case $(p \neq 0.5)$. This will be illustrated in Fig. 19 below. At the same time we should emphasize that we are now considering a situation where the coupling takes place via the functional values of the map $f\left(x_{k-1}\right)$ rather than via the argument $x_{k-1}$. This distinction is sometimes referred to as "nonlinear" rather than "linear" coupling. Finally, to relate the observed phenomena to previous work on chaotic synchronization, ${ }^{17}$ we shall take the coupling parameter $\varepsilon$ to be negative. With these conditions we again observe the formation of multilayered tori, but the mechanisms of destruction of these tori differ from the scenarios, that we observed for the map (1).

Figure 17(a) shows a chart of the dynamical modes in the parameter plane $(p, a)$ for the two-cluster system (2) with $\varepsilon=-1.4$. The domain of stability for period-2 cycle is denoted as $\Pi_{2}$. This domain is bounded to both sides by a curve 


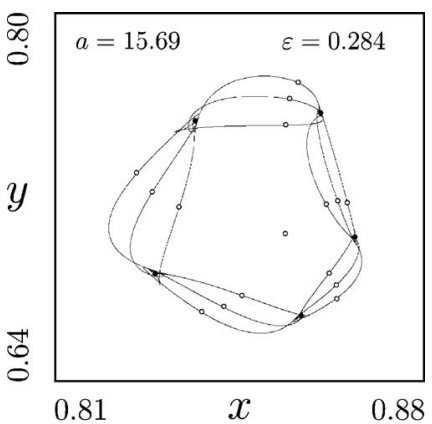

(a)

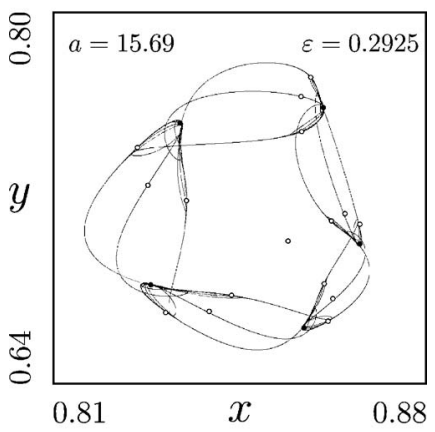

(b)

FIG. 15. Torus destruction through a homoclinic bifurcation. (a) Phase portrait of the map before the first homoclinic tangency. (b) Phase portrait immediately after the homoclinic tangency.

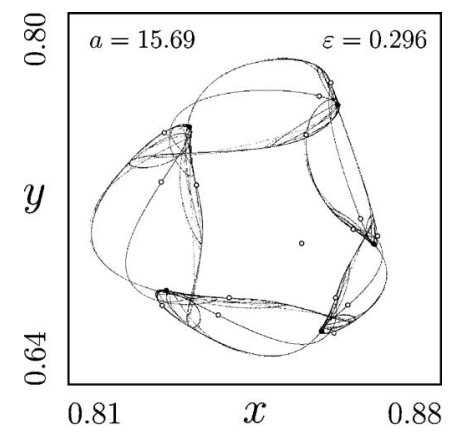

(a)

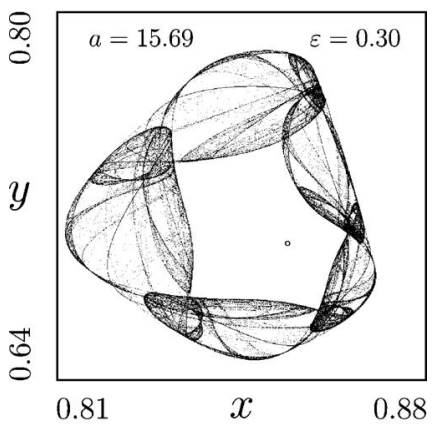

(b)

FIG. 16. Torus destruction through the homoclinic bifurcation. (a) Phase portrait of the map after the external layers have been destroyed. The middle layer continues to exist. (b) Phase portrait of the map after destruction of the middle layer.

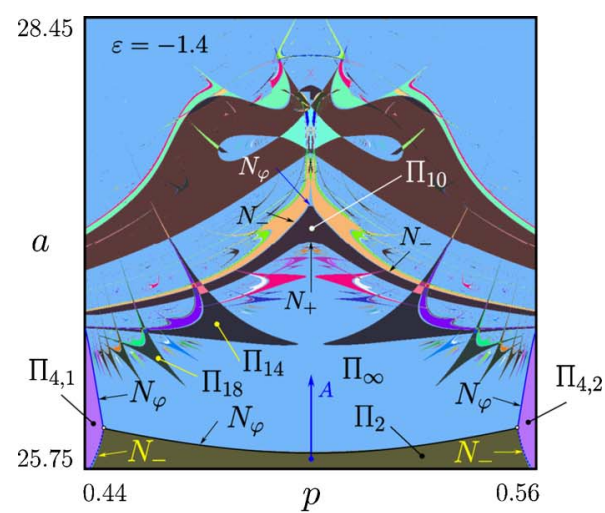

(a)

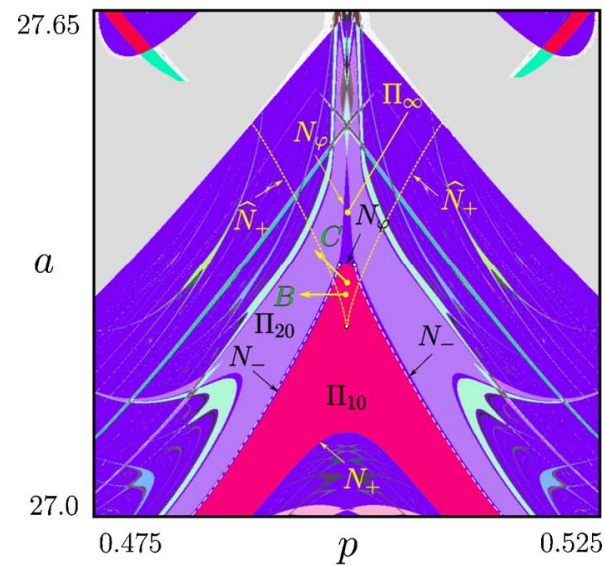

(b)

FIG. 17. (Color online) (a) Chart of the dynamical modes in the parameter plane $(p, a), \varepsilon=-1.4 . \Pi_{2}$ is the domain of existence of the stable period-2 cycle. $\Pi_{4,1}$ and $\Pi_{4,2}$ are the domains where the stable period-4 cycle exist. $\Pi_{10}, \Pi_{14}$, and $\Pi_{18}$ are the regions of period-10, period14 , and period-18 resonant dynamics, respectively. $N_{\varphi}$ is the NeimarkSacker bifurcation boundary and $\Pi_{\infty}$ is the domain of quasiperiodicity. $N_{+}$ denotes a saddle-node bifurcation curve and $N_{-}$is a period-doubling bifurcation curve. (b) Chart of the dynamical modes near the top of the resonance tongue $\Pi_{10} . \hat{N}_{+}$is the saddle-node bifurcation curve where the unstable node period-10 cycle $R$ merges with the saddle period-10 cycle and disappears. $\Pi_{20}$ is the domain of existence of the stable period- 20 cycle and $\Pi_{\infty}$ is the "triangle of quasiperiodicity," bounded by the three Neimark-Sacker bifurcation curves $N_{\varphi}$.

$N_{-}$of a period-doubling bifurcation. The upper boundary of the domain $\Pi_{2}$ is the Neimark-Sacker bifurcation curve $N_{\varphi}$ were an invariant torus arises from the stable period-2 cycle. The domain $\Pi_{\infty}$ in Fig. 17 is the region of quasiperiodicity. Within this region one can see a large number of periodic windows (e.g., $\Pi_{10}, \Pi_{14}, \Pi_{18}$ ) corresponding to various resonance modes.

Let us start our bifurcation analysis by moving from the region $\Pi_{2}$ into the region of quasiperiodicity along the direction $A$. Figure 18 shows a bifurcation diagram for the twocluster system with $p=0.5$. Within the region $\Pi_{2}$ the system has a single stable period- 2 cycle. On the line $N_{\varphi}$ the period-2 cycle undergoes a Neimark-Sacker bifurcation. With further increase of the value of parameter $a$, the system enters an Arnold tongue $\Pi_{10}$ with stable period-10 dynamics. This produces a one-layered resonance torus. Right after its formation, the stable period- 10 cycle is a node. 


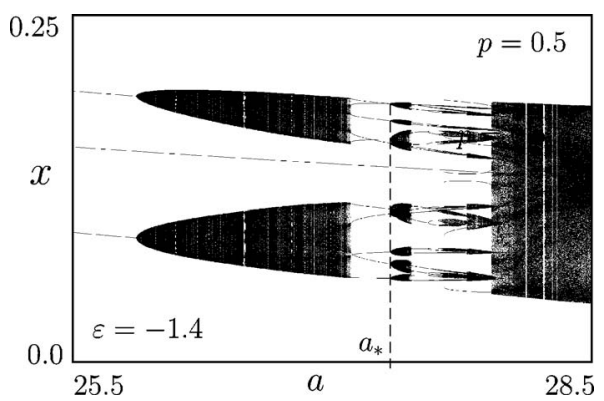

FIG. 18. Bifurcation diagram showing the transition from a stable period-2 cycle to a quasiperiodic orbit in a Neimark-Sacker bifurcation. The generated torus has two branches, one on either side of the diagonal. $a_{*}$ is the point of the secondary Neimark-Sacker bifurcation.

Note that the boundary of the resonance tongue $\Pi_{10}$, similar to the boundary of region $\Pi_{2}$ [see Fig. 17(b)], consists of three different bifurcation curves. The upper boundaries of the left and right wings denoted by $N_{-}$, are curves at which the period-10 cycle undergoes a period-doubling bifurcation, and the narrow middle part $N_{\varphi}$ is a curve of Neimark-Sacker bifurcation for the stable resonance cycle. The lower boundary of $\Pi_{10}$ is the saddle-node bifurcation curve $N_{+}$.

When the system leaves the resonance tongue $\Pi_{10}$ through the boundary $N_{\varphi}$, the stable node period-10 cycle turns into a stable focus to soon undergo a secondary Neimark-Sacker bifurcation and become an unstable focus. Before this happens, however, the one-layered torus has turned into a three-layered torus through a transverse pitchfork bifurcation of the saddle cycle. Figures 18, 19(a), and 19(b) allow us to follow the changes in the system's dynamics and phase portrait during these transformations. In particular, Fig. 19 illustrates the formation of the three-layered torus. Figure 19(a) presents the phase portrait immediately above the saddle-node bifurcation curve $N_{+}$where the twocluster system (2) displays an ordinary one-layered torus. In this figure, $F_{0}$ denotes the unstable nearly symmetric period-2 focus cycle.

As $a$ increases, the period-10 saddle cycle undergoes a transverse pitchfork bifurcation, and a three-layered torus is formed [see Fig. 19(b)]. In the asymmetric case, $p=0.4995$, the pitchfork bifurcation is replaced by a saddle-node bifurcation in which the unstable node $R$ and the saddle cycle $S_{1}$ are simultaneously born on the curve $\hat{N}_{+}$[Fig. 17(b)]. The result of this alternative route to the formation of a threelayered torus is shown in Fig. 19(c). We note the similarity with the phase portrait in Fig. 19(b), but also the lack of symmetry between the two saddle cycles $S_{1}$ and $S_{2}$.

Figure 20 shows the phase portrait after the second Neimark-Sacker bifurcation. This figure again refers to the symmetric case $p=0.5$. The period- 10 focus has now turned unstable and is surrounded by a region of quasiperiodic dynamics while both the inner and the outer layers of the threelayered torus continue exist. Figure 20(a) provides an overview of the full structure, while Figs. 20(b) and 20(c)

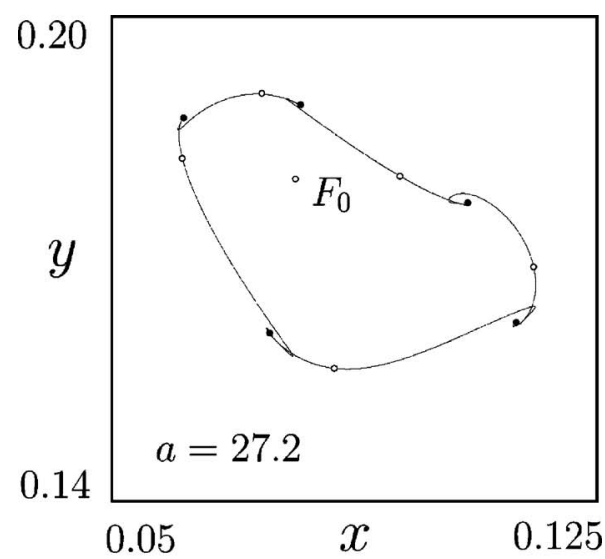

(a)

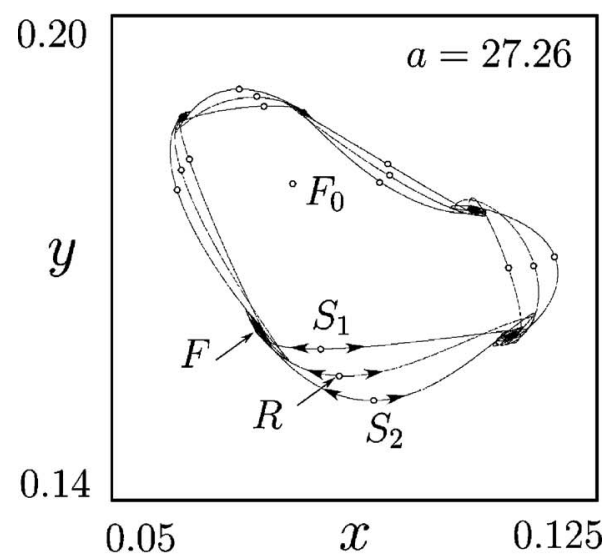

(b)

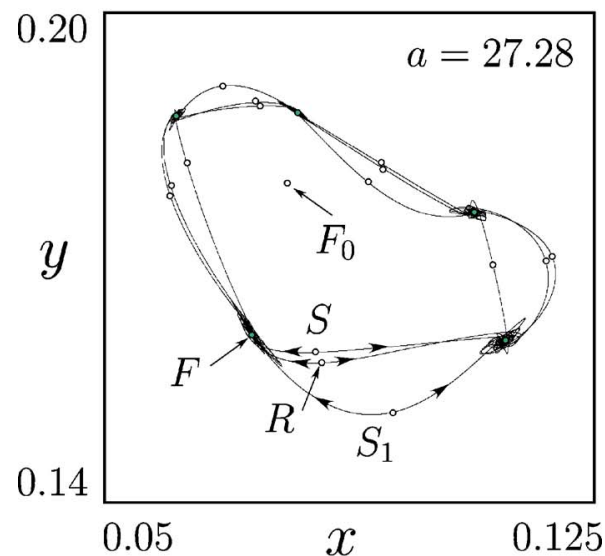

(c)

FIG. 19. (Color online) Birth of the three-layered period-10 resonance torus as the nonlinearity parameter $a$ is varied. (a) Phase portrait of the map before the pitchfork bifurcation, $p=0.5$ and $a=27.2$. (b) Three-layered resonance torus, $p=0.5$ and $a=27.26$. Here $F_{0}$ is the asymmetric unstable period-2 focus cycle, $F$ is the stable period-10 focus resonance cycle, $S_{1}$ and $S_{2}$ are the saddle period-10 cycles, $R$ is the unstable node period- 10 cycle (the repeller). (c) Phase portrait of the map for $p=0.4995$ and $a=27.28$. In this case $R$ and $S_{1}$ arise in a saddle-node bifurcation rather than in the pitchfork bifurcation that occurs between (a) and (b).

illustrate the local behavior of the middle-layer manifold and of one of the outer-layer manifolds, respectively.

We have observed three different ways for the destruction of the three-layered torus in our two-cluster system: 


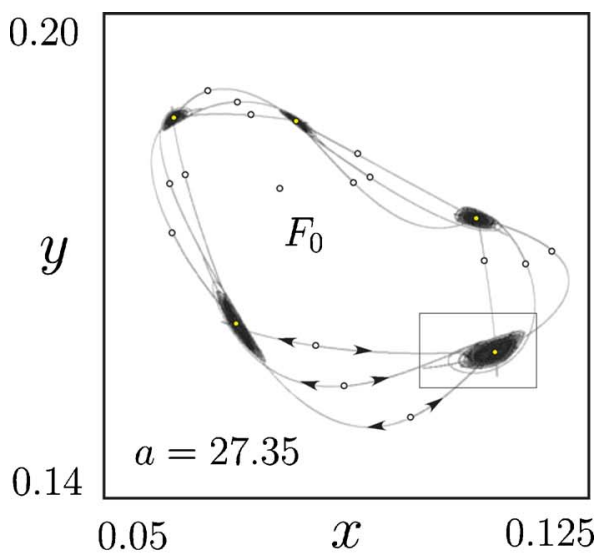

(a)

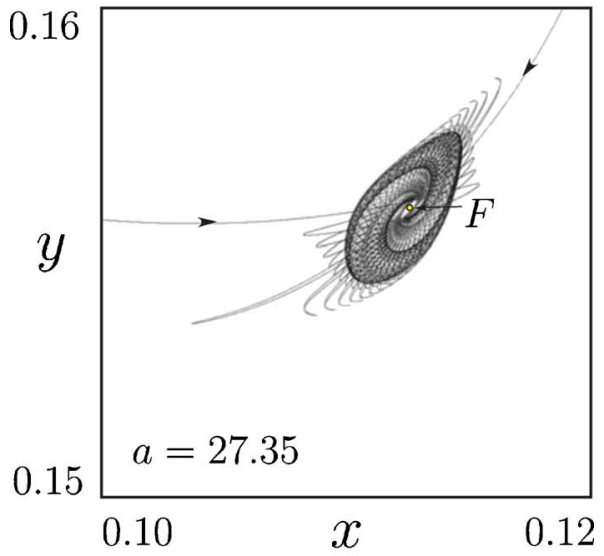

(b)

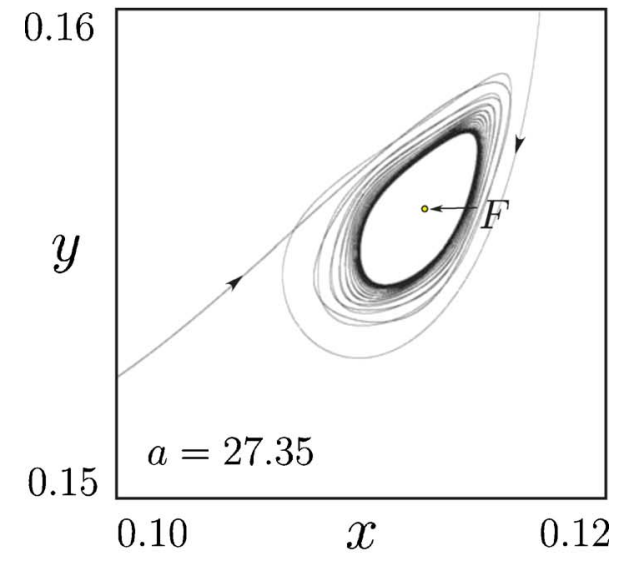

(c)

FIG. 20. (Color online) Secondary Neimark-Sacker bifurcation at the point $a=a_{*}$ in Fig. 18. (a) Phase portrait of the map (2) after the secondary Neimark-Sacker bifurcation. $F$ is the unstable period-10 focus cycle. (b) Magnified part of the phase portrait outlined by the rectangle in (a) showing one of the external layers. (c) The middle layer of three-layered torus, $p=0.5$.

In the first case, the three-layered torus is destroyed through a saddle-node bifurcation when we leave the resonance tongue $\Pi_{10}$ along the direction $B$ [see Fig. 17(b)]. First, the unstable node period-10 cycle $R$ and the saddle cycle $S$ merge and disappear in a saddle-node bifurcation on the line $\hat{N}_{+}$. This bifurcation leads to the disappearance of the middle and one external layer of the three-layered torus.

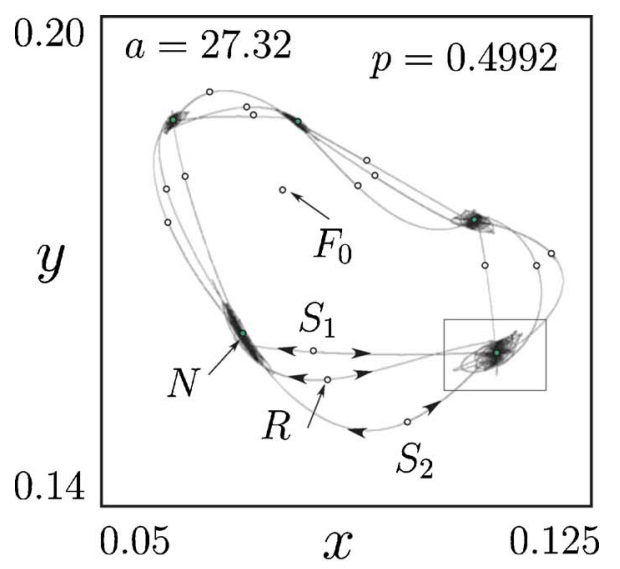

(a)

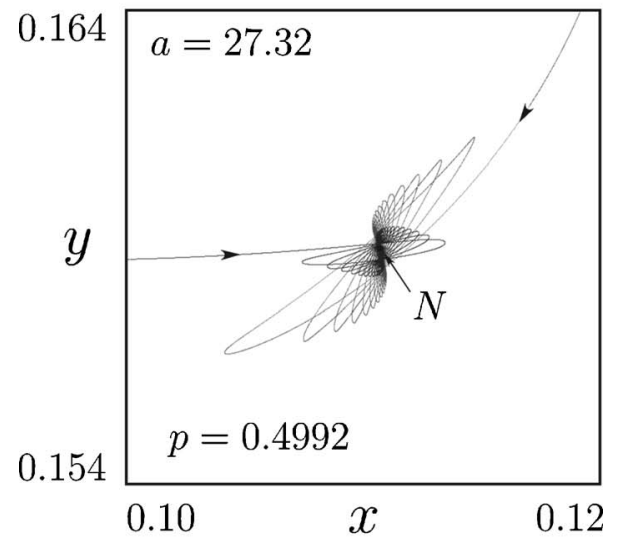

(b)

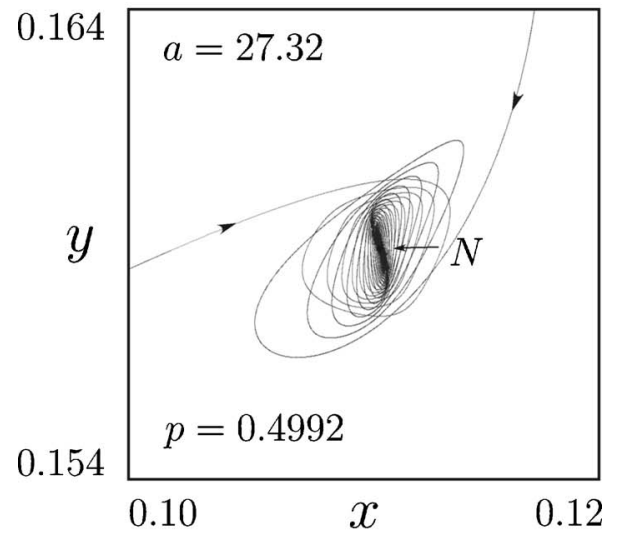

(c)

FIG. 21. (Color online) (a) Phase portrait of the map before the perioddoubling bifurcation when the stable period-10 focus cycle $F$ turns into a stable node period-10 cycle $N$ with negative multipliers. $a=27.32$ and $p$ $=0.4992$. (b) Magnified part of the phase portrait outlined by the rectangle in (a) showing the external layer. (c) The middle layer of three-layered torus. The nonleading manifold of the unstable node $R$ cycle winds around the stable node period-10 cycle instead of approaching it smoothly.

With further change of parameters along the direction $B$, the one-layered torus is destroyed in accordance with the classic scenario through a cascade of period-doubling bifurcations of the stable resonance cycle.

The second case describes how the three-layered torus is destroyed as we follow the direction $C$ from the domain $\Pi_{10}$ into the region $\Pi_{20}$. Figures 21 and 22 show phase portraits 


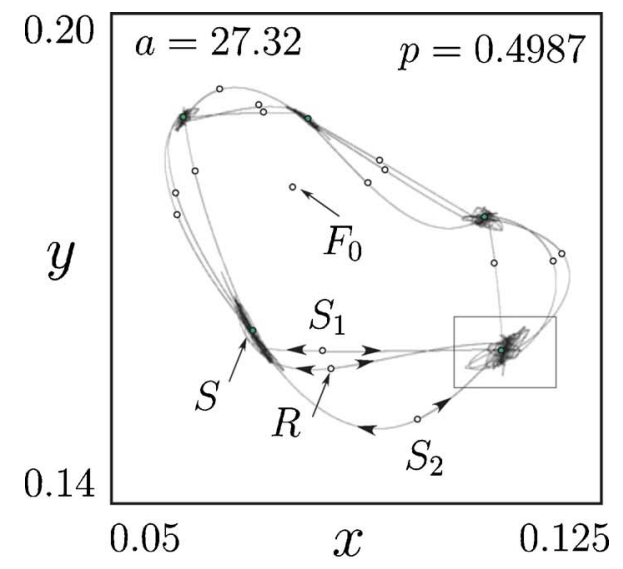

(a)

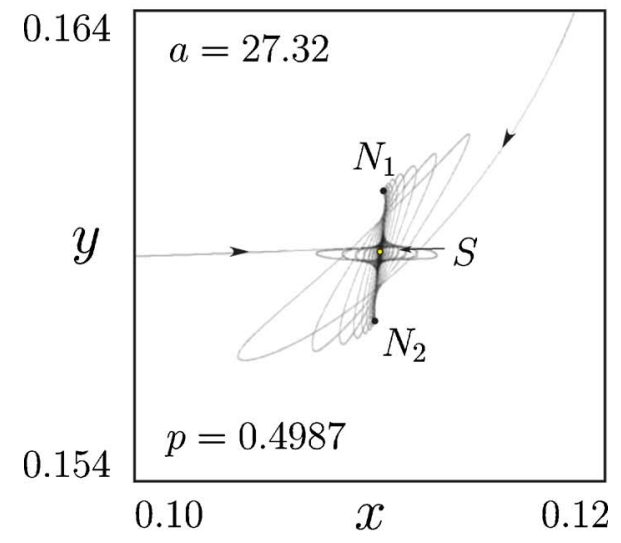

(b)

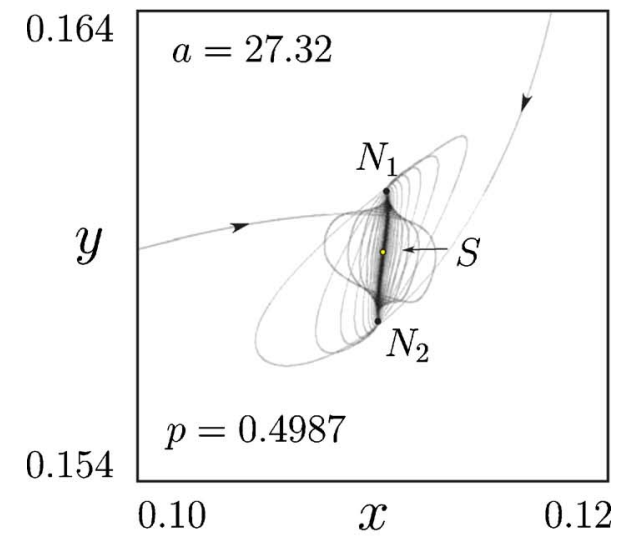

(c)

FIG. 22. (Color online) (a) Phase portrait of the map after the perioddoubling bifurcation, $p=0.4987$ and $a=27.32$. (b) Magnified part of the phase portrait outlined by the rectangle in (a). Local behavior one of the external layer manifolds. (c) The middle layer manifold. $N_{1}$ and $N_{2}$ are the points of the stable period- 20 node cycle.

illustrating the main stages of this process. First, the stable period-10 focus cycle $F$ [see Figs. 19(b) and 19(c)] turns into a stable node $N$ with negative multipliers. As illustrated by Figs. 21(b) and 12(c), the external layers contain loops, and the middle-layer manifold winds around the stable node period-10 point $N$ instead of approaching it in a more uniform manner. With further variation of the parameters, the period-10 cycle loses its stability as one of the multipliers leaves the unit circle through -1 , and a cycle with twice the

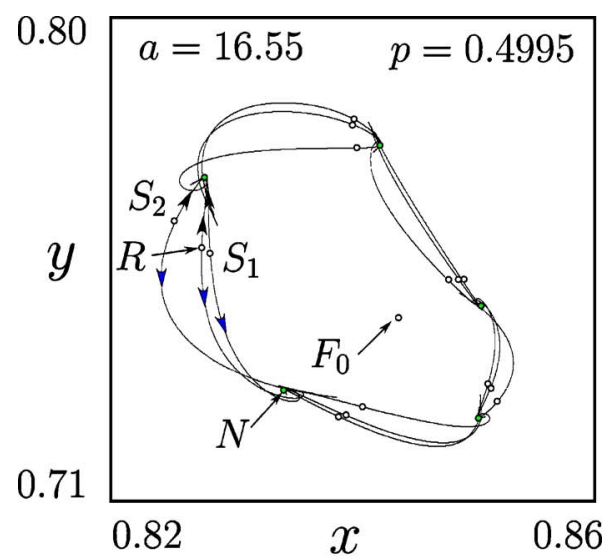

(a)

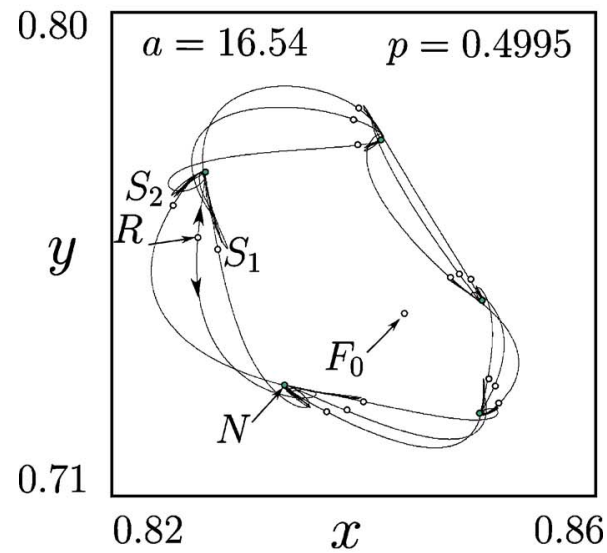

(b)

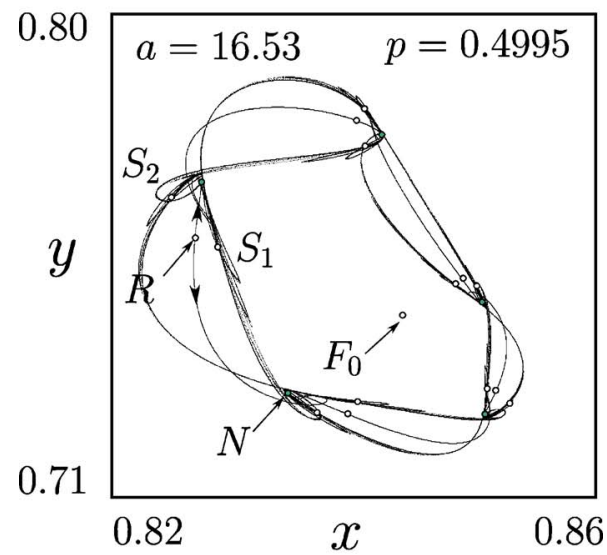

(c)

FIG. 23. (Color online) Torus destruction through a homoclnic bifurcation. (a) Phase portrait of the map before the homoclinic tangency. $a=16.55, p$ $=0.4995$, and $\varepsilon=-0.8$. (b) Phase portrait of the map near the homoclinic tangency. $a=16.54, p=0.4995$, and $\varepsilon=-0.8$. (c) Phase portrait of the map after the external layers have been destroyed through a homoclinic bifurcation. The middle layer continues to exist. $a=16.53, p=0.4995$ and $\varepsilon=-0.8$.

period arises (Fig. 22). Here we can observe the transition to chaos through an infinite sequence of period-doubling bifurcations.

In the third case the three-layered torus is destroyed through a homoclinic bifurcation. Figure 23(a) shows the phase portrait of the map before the homoclinic bifurcation. The external layers of this torus are formed by the saddle- 
node connection. First, two external layers are destroyed in a homoclinic bifurcation [Figs. 23(b) and 23(c)], and as the parameter $a$ decreases the middle layer is destroyed in a similar manner.

\section{CONCLUSION}

The problem of torus destruction was first discussed in the classic paper by Afraimovich and Shilnikov. ${ }^{5}$ They outlined three possible routes for the breakdown of a twodimensional torus. For all scenarios the starting point was a smooth torus in a resonance region where a stable periodic orbit (a node) coexists with an unstable orbit (a saddle cycle) of the same periodicity.

In one scenario, the unstable manifold from the saddle cycle begins to develop wrinkles as it approaches the points of the node. Hence, the torus becomes nonsmooth in these points. Under variation of a parameter, as the point of operation leaves the resonance zone, nonsmoothness spreads along the invariant manifold, and the torus breaks up into a fractal set. In the second scenario, the unstable manifold from the saddle cycle starts to intersect the nonleading manifold of the node. This again produces an infinitely folded structure, and the torus breaks down when this structure makes contact to the stable manifold of the same saddle cycle. Finally, the stable node may transform into a stable focus to subsequently undergo a sequence of period-doubling bifurcations. This again leads to breakdown of the torus. The above scenarios apply to systems that can be described by twodimensional invertible maps.

Some of the modifications that arise for noninvertible maps were discussed in previous publications. ${ }^{6-9}$ The absence of invertibility allows the manifolds to intersect themselves, and for all of the above scenarios the characteristic feature of noninvertible systems is that the wrinkling of the manifold from the saddle cycle proceeds through the appearance of an infinite series of cusp points and thereafter a series of self-intersecting loops. ${ }^{10}$

In the present paper we considered a further extension to the existing picture for noninvertible maps. We showed that a two-dimensional map, representing either two coupled intermittency I maps or the two-cluster dynamics of an ensemble of a large number of globally coupled intermittency I maps can lead to the formation of multilayered tori.

We first demonstrated how multilayered tori in the symmetric case arise in ordinary pitchfork or period-doubling bifurcations transverse to the torus surface, and that they can be observed for both linear and so-called nonlinear coupling. In the asymmetric case, that is of relevance particularly to the cluster formation in ensembles of many oscillators, the pitchfork bifurcation is replaced by a saddle-node bifurcation.

Next, we described three different scenarios for the breakdown of the multilayered tori in a system of two symmetrically coupled intermittency maps. Although all of these scenarios have aspects in common with the classic Afraimovich-Shilnikov scenarios, they are clearly different in their details. It is possible, for instance, that the inner layer in a multilayered torus can disappear in a saddle-node bifurcation while the external layers form closed-loop structures of stable and unstable cycles. It is also possible that homoclinic bifurcations that destroy the torus can occur separately for the inner and outer layers.

We have previously discussed the formation and destruction of a bilayered torus in connection with the analysis of a piecewise-smooth model of a power electronic $\mathrm{dc} / \mathrm{dc}$ converter. ${ }^{12}$ The bilayered torus observed in that case consisted of the closure of a stable focus cycle with two saddle cycles and their connecting manifolds, and the saddle cycles, of which one had a single and the other two unstable directions, arose through a pair of coinciding saddle-node and border-collision bifurcations. We conclude that multilayered tori may also be observed in time-continuous systems.

\section{ACKNOWLEDGMENTS}

The work was supported by the Russian Foundation for Basic Research Grant No. 06-01-00811, and by the EU Network of Excellence BioSim Contract No. LSHB-CT-2004005137.

${ }^{1}$ R. V. Buskirk and C. Jeffries, Phys. Lett. 31A, 3332 (1985).

${ }^{2}$ J. Frøyland, Physica D 8, 423 (1983).

${ }^{3}$ V. S. Anishchenko, Dynamical Chaos in Physical Systems (Teubner-Texte zur Physik, Leipzig, 1999).

${ }^{4}$ C. Reick and E. Mosekilde, Phys. Rev. E 52, 1418 (1995).

${ }^{5}$ V. S. Afraimovich and L. P. Shilnikov, Am. Math. Soc. Transl. 149, 201 (1991).

${ }^{6}$ C. E. Frouzakis, L. Gardini, I. G. Kevrekidis, G. Millerioux, and C. Mira, Int. J. Bifurcation Chaos Appl. Sci. Eng. 7, 2101 (1997).

${ }^{7}$ C. E. Frouzakis, I. G. Kevrekidis, and B. B. Peckhman, Physica D 177, 101 (2003).

${ }^{8}$ E. Sander, Nonlinear Anal. Theory, Methods Appl. 41, 259 (2000).

${ }^{9}$ R. Rico-Martinez, R. A. Adomaitis, and I. G. Kevrekidis, Comput. Chem. Eng. 24, 2417 (2000)

${ }^{10}$ V. Maistrenko, Y. Maistrenko, and E. Mosekilde, Phys. Rev. E 67, 046215 (2003).

${ }^{11}$ Zh. T. Zhusubaliyev and E. Mosekilde, Bifurcations and Chaos in Piecewise-Smooth Dynamical Systems (World Scientific, Singapore, 2003).

${ }^{12}$ Zh. T. Zhusubaliyev and E. Mosekilde, Phys. Lett. A 351, 167 (2006).

${ }^{13}$ P. Manneville and Y. Pomeau, Physica D 1, 219 (1980).

${ }^{14}$ Y. Pomeau and P. Manneville, Commun. Math. Phys. 74, 189 (1980).

${ }^{15}$ L. Glass, M. R. Shrier, and R. Perez, Physica D 7D, 89 (1983).

${ }^{16}$ D. Parthimos, D. H. Edwards, and T. M. Griffith, Phys. Rev. E 64, 061906 (2001).

${ }^{17}$ M. Manscher, M. Nordahn, E. Mosekilde, and Yu. L. Maistrenko, Phys Lett. A 238, 358 (1998).

${ }^{18}$ E. Ott and J. C. Sommerer, Phys. Lett. A 188, 39 (1994).

${ }^{19}$ P. Aswin, J. Buescu, and I. Stewart, Nonlinearity 9, 703 (1996).

${ }^{20}$ Yu. L. Maistrenko, V. L. Maistrenko, O. Popovich, and E. Mosekilde, Phys. Rev. E 60, 2817 (1999).

${ }^{21}$ A. Agliari, G. I. Bischi, R. Dieci, and L. Gardini, Int. J. Bifurcation Chaos Appl. Sci. Eng. 15, 1285 (2005).

${ }^{22}$ K. Kaneko, Phys. Rev. Lett. 17, 219 (1989).

${ }^{23}$ K. Kaneko, Physica D 41, 137 (1990).

${ }^{24}$ E. Mosekilde, Yu. Maistrenko, and D. Postnov, Chaotic Synchronization: Applications to Living Systems (World Scientific, Singapore, 2002).

${ }^{25}$ K. Kaneko, Physica D 23, 436 (1986).

${ }^{26}$ D. Noble, A. Varghese, P. Kohl, and P. J. Noble, Can. J. Cardiol. 14, 123 (1998).

${ }^{27}$ R. M. Santos, L. M. Rosario, A. Nadal, J. Garcia-Sancho, B. Soria, and M. Valdeolmillos, Pfluegers Arch. 418, 417 (1991).

${ }^{28}$ P. Tass, Phys. Rev. E 56, 2043 (1997). 